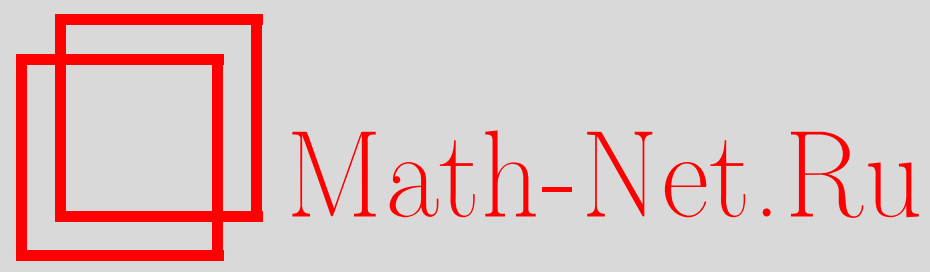

С. Ф. Лукомский, О сходимости рядов Уолша в пространствах, близких к $L^{\infty}$, Матем. заметки, 2001, том 70, выпуск $6,882-889$

DOI: https://doi.org/10.4213/mzm800

Использование Общероссийского математического портала Math-Net.Ru подразумевает, что вы прочитали и согласны с пользовательским соглашением http://www . mathnet.ru/rus/agreement

Параметры загрузки:

IP: 52.205 .19 .152

26 апреля 2023 г., $12: 36: 37$

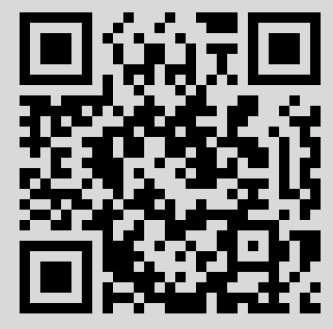




\section{О СХОДИМОСТИ РЯДОВ УОЛША \\ В ПРОСТРАНСТВАХ, БЛИЗКИХ К $L^{\infty}$}

\section{С. Ф. Лукомский}

Рассматриваются пространства, близкие к $L^{\infty}$, которые являются объединением классов Орлича. Доказывается сходимость рядов Уолша в полученных пространствах.

Библиографоял: 3 названия.

1. Введение. Хорошо известно [1, с. 124], что если $f \in L_{p}(1<p<\infty)$, то ее ряд Фурье-Уолша сходится по норме $L_{p}$. Ситуация меняется, если $f$ принадлежит пространствам, лежашим между $L_{1}$ и $L_{p}$ или между $L_{p}$ и $L^{\infty}$. Так в работе [2] была доказана эквивалентность следующих утверждений:

i) для любой непрерьвной функции $f$ ее ряд Фурье-Уолша сходится в пространстве $L(\varphi)$;

ii) $L\left(e^{x^{2}}\right) \subset L(\varphi)$.

Мы рассмотрим этот вопрос для случая пространств, лежащих по шкалепространств Орлича между $L_{p}$ и $L^{\infty}$. В качестве таких пространств рассмотрим объединение классов Орлича с нормой, отличной от норм Орлича и Люксембурга.

2. Функциональные пространства, близкие к $L^{\infty}$. Пусть $(G, \Sigma, \mu)-$ пространство с конечной мерой $\mu$ и $\mu(G)=1$. Классы Орлича $L^{o}(\varphi)$ в случае, когда $N$-функция $\varphi$ не удовлетворяет $\Delta_{2}$-условию, не являются линейными. Чтобы превратить такой класс в линейное пространство, рассматривают их объединение $L(\varphi)=\bigcup_{\lambda} L^{o}(\varphi(\cdot \lambda))$ и традиционно определяют норму одним из следующих равенств:

$$
\begin{aligned}
& \|f\|_{1}=\sup \left\{\int_{G}|f g| d \mu: \int_{G} \varphi^{*}(g) d \mu \leqslant 1\right\} \quad \text { (норма Орлича), } \\
& \|f\|_{2}=\inf \left\{\lambda>0: \int_{G} \varphi\left(\frac{|f|}{\lambda}\right) d \mu \leqslant 1\right\} \quad \text { (норма Люксембурга). }
\end{aligned}
$$

Мы рассмотрим пространства, лежащие по шкалепространств Орлича между $L_{p}$ и $L^{\infty}$, определим в них норму, отличную от норм Орлича и Люксембурга, и покажем, что полученные пространства являются сепарабельными банаховыми пространствами.

Работа выполнена при поддержке программы “Ведущие научные школы”, грант № 00-15-96123. 
ОПРЕДЕЛЕНИЕ. Пусть $p>1, \alpha \geqslant 1$. Через $\widehat{\mathscr{L}}_{p, \alpha}(G)$ обозначим совокупность всех измеримых, почти всюду (п.в.) конечных функций $f: G \rightarrow \mathbb{R}$ таких, что для каждой $f \in \widehat{\mathscr{L}}_{p, \alpha}(G)$

1) существует положительная, непрерьвная, строго возрастающая на $(0, \infty)$ функция $\varphi$ такая, что

$$
\int_{1}^{\infty}\left(\frac{\varphi^{-1}(x)}{x^{\alpha}}\right)^{p} d x<\infty \quad\left(\varphi^{-1}-\text { обратная к } \varphi\right),
$$

2) существует постоянная $\gamma>1$ такая, что

$$
\int_{G} \gamma^{\varphi(|f|)} d \mu<\infty
$$

Tеорема 1. Множество $\widehat{\mathscr{L}}_{p, \alpha}(G)$ есть банахово пространство с нормой

$$
\|f\|_{\hat{p}, \alpha}=\left(\int_{1}^{\infty}\left(\frac{\|f\|_{x}}{x^{\alpha}}\right)^{p} d x\right)^{1 / p}<\infty
$$

әде $\|\cdot\|_{x}-$ норма в пространстве $L_{x}$.

ДокАЗАтЕльСтво. Покажем, что $\widehat{\mathscr{L}}_{p, \alpha}(G)$ совпадает с множеством функций, для которых конечна величина

$$
\sum_{n=1}^{\infty}\left(\frac{\|f\|_{n}}{n^{\alpha}}\right)^{p}
$$

1) Пусть $f$ измерима, п.в. конечна и ряд (4) сходится. Покажем, что существует непрерьвная, строго возрастающая функция $\varphi(x)>0$ такая, что интеграл (2) сходится при некотором $\gamma>1$ и сходится интеграл (1).

Если $|f(x)|=$ const $=A$ п.в. на $G$, то в качестве $\varphi^{-1}$ можно взять любую положительную, непрерывную, строго возрастающую функцию такую, что

$$
\lim _{x \rightarrow \infty} \varphi^{-1}(x)=B>A .
$$

Если $|f(x)| \neq$ const п.в. на $G$, то для $\lambda>1$ положим

$$
\varphi^{-1}(x)= \begin{cases}\lambda\|f\|_{x} & \text { при } x>1, \\ \lambda x\|f\|_{1} & \text { при } 0<x \leqslant 1 .\end{cases}
$$

Тогда $\varphi^{-1}(x)$ строго возрастает, непрерьвна на $(0,+\infty)$ и, значит, существует обратная к ней $\varphi$. Очевидно, что интеграл (1) сходится.

Покажем, что интеграл (2) сходится при $1<\gamma<\lambda$. Пусть

$$
E_{n}=\{x: n \leqslant \varphi(|f(x)|)<n+1\} .
$$

Тогда

$$
\|f\|_{n}^{n}=\sum_{k=0}^{\infty} \int_{E_{k}}|f|^{n} d \mu \geqslant \int_{E_{n}}|f|^{n} d \mu \geqslant\left(\varphi^{-1}(n)\right)^{n} \mu E_{n}
$$


откуда находим, что при $1<\gamma<\lambda$ вьполнена оценка

$$
\begin{aligned}
\int_{G} \gamma^{\varphi(|f|)} d \mu & \leqslant \gamma \sum_{k=0}^{\infty} \gamma^{k} \mu E_{k} \leqslant \gamma \sum_{k=1}^{\infty} \gamma^{k} \frac{\|f\|_{k}^{k}}{\left(\varphi^{-1}(k)\right)^{k}}+\gamma \mu E_{0} \\
& =\gamma \mu E_{0}+\gamma \sum_{k=1}^{\infty}\left(\frac{\gamma}{\lambda}\right)^{k}<\infty
\end{aligned}
$$

2) Покажем обратное включение. Пусть при некотором $\gamma>1$ интегралы (1) и (2) сходятся. Снова определим множества $E_{n}$ равенствами (5). Тогда

$$
\sum_{n=0}^{\infty} \gamma^{n} \mu E_{n} \leqslant \int_{G} \gamma^{\varphi(|f|)} d \mu \leqslant \gamma \sum_{n=0}^{\infty} \gamma^{n} \mu E_{n}
$$

и для нормы $\|f\|_{n}$ имеем

$$
\|f\|_{n}^{n} \leqslant \sum_{k=0}^{\infty}\left(\varphi^{-1}(k+1)\right)^{n} \mu E_{k}=\gamma \sum_{k=0}^{\infty} \gamma^{k} \mu E_{k} \frac{\left(\varphi^{-1}(k+1)\right)^{n}}{\gamma^{k+1}}
$$

или

$$
\|f\|_{n} \leqslant \gamma^{1 / n}\left(\sum_{k=0}^{\infty} \gamma^{k} \mu E_{k}\right)^{1 / n} \sup _{k} \frac{\varphi^{-1}(k+1)}{\gamma^{(k+1) / n}} .
$$

Обозначим

$$
A=\sup _{n} \gamma^{1 / n}\left(\sum_{k=0}^{\infty} \gamma^{k} \mu E_{k}\right)^{1 / n}
$$

и пусть $k_{n}$ есть то значение $k+1$, при котором достигается $\operatorname{sup~в~}(7)$. Тогда (7) примет вид

$$
\|f\|_{n} \leqslant A \frac{\varphi^{-1}\left(k_{n}\right)}{\gamma^{k_{n} / n}}
$$

откуда находим

$$
\sum_{n=1}^{\infty}\left(\frac{\|f\|_{n}}{n^{\alpha}}\right)^{p} \leqslant A^{p} \sum_{n=1}^{\infty}\left(\frac{\varphi^{-1}\left(k_{n}\right)}{\gamma^{k_{n} / n}}\right)^{p} \frac{1}{n^{\alpha p}} .
$$

Покажем, что ряд справа в (8) сходится. Обозначим

$$
K(s)=\left\{n: s \leqslant \frac{k_{n}}{n}<s+1\right\} \quad(s=0,1, \ldots) .
$$

Тогда

$$
\begin{aligned}
\sum_{n=1}^{\infty}\left(\frac{\varphi^{-1}\left(k_{n}\right)}{\gamma^{k_{n} / n}}\right)^{p} \frac{1}{n^{\alpha} p} & =\sum_{s} \sum_{n \in K(s)}\left(\frac{\varphi^{-1}\left(k_{n}\right)}{\gamma^{k_{n} / n}}\right)^{p} \frac{1}{n^{\alpha p}} \\
& \leqslant \gamma^{p} \sum_{s} \frac{(s+1)^{\alpha p}}{\gamma^{(s+1) p}} \sum_{n \in K(s)}\left(\frac{\varphi^{-1}(n(s+1))}{(n(s+1))^{\alpha}}\right)^{p} \\
& \leqslant \gamma^{p} \sum_{s} \frac{(s+1)^{\alpha p}}{\gamma^{(s+1) p}} \sum_{n=1}^{\infty}\left(\frac{\varphi^{-1}(n)}{n^{\alpha}}\right)^{p}<\infty
\end{aligned}
$$


Таким образом, множество $\widehat{\mathscr{L}}_{p, \alpha}(G)$ совпадает с множеством всех функций, для которых сходится ряд (4).

3) Очевидно, что $\widehat{\mathscr{L}}_{p, \alpha}(G)$ является линейньм пространством и любое из равенств

$$
\|f\|_{\hat{p}, \alpha}=\left(\sum_{n=1}^{\infty}\left(\frac{\|f\|_{n}}{n^{\alpha}}\right)^{p}\right)^{1 / p}, \quad\|f\|_{\hat{p}, \alpha}=\left(\int_{1}^{\infty}\left(\frac{\|f\|_{x}}{x^{\alpha}}\right)^{p} d x\right)^{1 / p}
$$

определяет в $\widehat{\mathscr{L}}_{p, \alpha}(G)$ эквивалентные нормы. Покажем полноту.

Во-первых, $\hat{\mathscr{L}}_{p, \alpha}(G)$ - идеальное пространство, т.е. норма $\|f\|_{\hat{p}, \alpha}$ монотонна.

Во-вторьх, норма $\|\cdot\|_{\hat{p}, \alpha}$ порядково непрерьвна, т.е. если последовательность $0 \leqslant$ $f_{n}(t) \downarrow 0$ п.в., то $\left\|f_{n}\right\|_{\hat{p}, \alpha} \rightarrow 0$. В самом деле, при любом $x \geqslant 1$ вьполнено неравенство $\left\|f_{n+1}\right\|_{x} \leqslant\left\|f_{n}\right\|_{x}$ и по теореме Лебега $\left\|f_{n}\right\|_{x} \rightarrow 0$. Еше раз применяя теорему Лебега, получаем, что $\left\|f_{n}\right\|_{\hat{p}, \alpha} \rightarrow 0$.

В-третьих, норма $\|\cdot\|_{\hat{p}, \alpha}$ порядково полна, т.е. из $0 \leqslant f_{n} \uparrow f$ и $\left\|f_{n}\right\|_{\hat{p}, \alpha} \leqslant M$ следует, что $f \in \widehat{\mathscr{L}}_{p, \alpha}(G)$ и $\|f\|_{\hat{p}, \alpha} \leqslant M$. В самом деле, так как $0 \leqslant f_{n} \uparrow f$, то

$$
\left(\frac{\left\|f_{n}\right\|_{x}}{x^{\alpha}}\right)^{p} \uparrow F(x)
$$

и по теореме Леви $F(x) \in L$ и для любого $x \geqslant 1$ вьполнена оценка $\left\|f_{n}\right\|_{x} \leqslant x^{\alpha} F^{1 / p}(x)$. Поэтому для любого $x$

$$
\int_{G}\left|f_{n}(t)\right|^{x} d \mu(t) \leqslant\left(x^{\alpha} F^{1 / p}(x)\right)^{x}
$$

и снова по теореме Леви $|f(t)|^{x}$ интегрируема по $d \mu(t)$, причем

$$
\int_{G}|f(t)|^{x} d \mu(t) \leqslant\left(x^{\alpha} F^{1 / p}(x)\right)^{x} .
$$

Отсюда по теореме Лебега и следует сходимость интеграла (3). Но идеальное пространство, в котором норма порядково непрерывна и порядково полна, будет полньп [3, c. 143]. Теорема доказана.

Укажем теперь, где расположены пространства $\widehat{\mathscr{L}}_{p, \alpha}(G)$ по шкале пространств Орлича.

Теорема 2. Пусть $p>1, q>0, \alpha \geqslant 1,1 / p+1 / q=\alpha$. Тогда для любого $\gamma>1 u$ любого $r>q$

$$
L^{o}\left(\gamma^{|x|^{r}}\right) \subset \widehat{\mathscr{L}}_{p, \alpha}(G) \subset L^{o}\left(\gamma^{|x|^{q}}\right) .
$$

ДокАЗАТЕЛЬСтво. Покажем левое включение. Выберем $f \in L^{o}\left(\gamma^{|x|^{r}}\right)$ и положим

$$
E_{n}=\left\{x: n \leqslant|f(x)|^{r}<n+1\right\} .
$$

Тогда

$$
\infty>A=\int_{G} \gamma^{|f|^{r}} d \mu \geqslant \sum_{k=0}^{\infty} \gamma^{k} \mu E_{k}
$$


С другой стороны,

$$
\|f\|_{n}^{n} \leqslant \sum_{k=0}^{\infty}(k+1)^{n / r} \mu E_{k}=\sum_{k=0}^{\infty} \gamma^{k} \mu E_{k} \frac{(k+1)^{n / r}}{\gamma^{k}} .
$$

Обычными методами анализа убеждаемся, что

$$
\sup _{k} \frac{(k+1)^{n / r}}{\gamma^{k}} \leqslant \gamma\left(\frac{n}{r \ln \gamma}\right)^{n / r} \gamma^{-n /(r \ln \gamma)} .
$$

Из (11), (12) и (13) находим

$$
\|f\|_{n} \leqslant A^{1 / n} C_{r, \gamma} n^{1 / r}
$$

и, значит,

$$
\sum\left(\frac{\|f\|_{n}}{n^{\alpha}}\right)^{p}<\infty
$$

при $r>q$.

Проверим правое включение. Выберем $f \in \widehat{\mathscr{L}}_{p, \alpha}(G)$ и положим

$$
E_{n}=\left\{x: n \leqslant|f(x)|^{q}<n+1\right\} .
$$

Тогда

$$
\int_{G} \gamma^{|f|^{r}} d \mu \leqslant \gamma \sum_{n=0}^{\infty} \gamma^{n} \mu E_{n}
$$

Покажем, что

$$
\lim _{n \rightarrow \infty}\left(\mu E_{n}\right)^{1 / n}=0
$$

Имеем

$$
\|f\|_{n}^{n}=\int_{G}|f|^{n} d \mu \geqslant \int_{E_{n}}|f|^{n} d \mu \geqslant n^{n / q} \mu E_{n}
$$

и, значит,

$$
\left(\mu E_{n}\right)^{1 / n} \leqslant \frac{\|f\|_{n}}{n^{1 / q}} .
$$

Так как $\|f\|_{n}$ возрастает, то сходимость ряда (14) равносильна сходимости ряда

$$
\sum_{n}\left(\frac{\|f\|_{2^{n}}}{2^{n \alpha}}\right)^{p} 2^{n}
$$

Поэтому

$$
\lim _{n \rightarrow \infty} \frac{\|f\|_{2^{n}}^{p}}{2^{n(\alpha p-1)}}=\lim _{n \rightarrow \infty}\left(\frac{\|f\|_{2^{n}}}{\left(2^{n}\right)^{1 / q}}\right)^{p}=0,
$$

откуда с учетом (17) и следует (16). Но тогда радиус сходимости ряда (15) равен $\infty$, т.е. ряд (15) сходится при всех $\gamma>1$.

3. Сходимость рядов Уолша в пространствах $\widehat{\mathscr{L}}_{p, \alpha}$. Рассмотрим функции Уолша $\left\{W_{n}(t)\right\}_{n=0}^{\infty}$ в нумерации Пэли на двоичной группе $G$. Мера $\mu E$ в этом случае совпадает с мерой Лебега $|E|$. 
Лемма. Ступенчатые функиии, постоянные на двоичных интервалах, образуют плотное в $\widehat{\mathscr{L}}_{p, \alpha}$ множество.

ДокАЗАтЕльство. Так как норма $\|f\|_{\hat{p}, \alpha}$ в $\widehat{\mathscr{L}}_{p, \alpha}$ порядково непрерывна, то функции $\sum_{k=1}^{n} \lambda_{k} \chi_{E_{k}}$, где $E_{k}$ - измеримые множества, образуют плотное в $\widehat{\mathscr{L}}_{p, \alpha}[3$, с.142] множество. Но каждое измеримое множество $E_{k}$ есть предел убьвающей последовательности множеств $\bigcup_{\nu=1}^{n} I_{k, \nu}$, где $I_{k, \nu}$ - двоичные интервалы. Поэтому из порядковой непрерьвности нормы следует утверждение леммы.

Отметим, что из доказанной леммы следует сепарабельность пространства $\widehat{\mathscr{L}}_{p, \alpha}$. В заключение докажем теорему о сходимости рядов Уолша в пространствах $\widehat{\mathscr{L}}_{p, \alpha}$.

ТЕОрема 3. Пусть $p>1, \alpha \geqslant 1, S_{m}(f)$ - частичные суммы ряда Фурье-Уолиа функиии $f$. Тогда

1) для любой $f \in \widehat{\mathscr{L}}_{p, \alpha}$

$$
\left\|S_{m}(f)\right\|_{\hat{p}, \alpha+1} \leqslant C_{p, \alpha}\|f\|_{\hat{p}, \alpha}
$$

2) для любой $f \in \widehat{\mathscr{L}}_{p, \alpha}\left\|S_{m}(f)-f\right\|_{\hat{p}, \alpha+1} \rightarrow 0$;

3) существует $f \in \widehat{\mathscr{L}}_{p, \alpha}$ такая, что нормы $\left\|S_{m}(f)\right\|_{\hat{p}, \alpha+1-\varepsilon}$ неограничены при всех $\varepsilon>0$.

ДокАЗАтЕльСтво. 1) Интерполяционная теорема Марцинкевича для частичных сумм $S_{m}(f)$ Фурье-Уолша при $1<r<2$ дает оценку

$$
\left\|S_{m}(f)\right\|_{r} \leqslant C\left(\frac{r}{(r-1)(2-r)}\right)^{1 / r}\|f\|^{r}=C_{r}\|f\|^{r} .
$$

Отсюда находим, что если $r$ и $r^{\prime}$ связаны соотношением $1 / r+1 / r^{\prime}=1$, то $[1$, c. 124$]$

$$
\left\|S_{m}(f)\right\|_{r^{\prime}} \leqslant C_{r}\|f\|_{r^{\prime}}
$$

Полагая $r^{\prime}=n \in \mathbb{N}, n>1$, получаем, что существует постоянная $C>0$ такая, что

$$
\left\|S_{m}(f)\right\|_{n} \leqslant C n\|f\|_{n},
$$

откуда сразу и следует утверждение 1$)$.

2) Так как ступенчатые функции (т.е. многочлены Уолша) образуют плотное в $\widehat{\mathscr{L}}_{p, \alpha}$ множество, то утверждение 2) обычным образом вытекает из (18).

3) Построим функцию $f \in \widehat{\mathscr{L}}_{p, \alpha}$, для которой нормы $\left\|S_{n}(f)\right\|_{\hat{p}, \alpha+1-\varepsilon}$ неограничены при всех $\varepsilon>0$. Выберем произвольную пока последовательность $m_{k} \uparrow \infty$, считая $m_{0}=0, m_{k+1}-m_{k} \geqslant 2, m_{k} \in \mathbb{N}$. Определим функцию $f$ на $G$ равенством

$$
f(x)=\lambda_{k} \quad \text { при } \quad x \in \Delta_{0}^{\left(m_{k-1}\right)} \backslash \Delta_{0}^{\left(m_{k}\right)}=\left[\frac{1}{2^{m_{k}}}+0, \frac{1}{2^{m_{k-1}}}-0\right] \quad(k=1,2, \ldots) .
$$

Числа $\lambda_{k}$ и $m_{k}$ подберем позже. При

$$
m=\sum_{j=1}^{s} \sum_{i=m_{2 j-1}}^{m_{2 j}-1} 2^{i}
$$


рассмотрим модифицированные частичные суммы [1, с. 120]

$$
S_{m}^{*}(f)=\int_{G} f(x \oplus t) D_{m}^{*}(t) d \mu(t)=\sum_{j=1}^{2 s} \frac{(-1)^{j}}{\left|\Delta_{x}^{\left(m_{j}\right)}\right|} \int_{\Delta_{x}^{\left(m_{j}\right)}} f(t) d \mu(t)
$$

где $\Delta_{x}^{(m)}$ означает двоичньй интервал ранга $m$, содержащий точку $x$. Найдем явное выражение для $S_{m}^{*}(f)$.

Если $x \in \Delta_{0}^{\left(m_{2 s}\right)}$, то $\Delta_{x}^{\left(m_{j}\right)}=\Delta_{0}^{\left(m_{j}\right)}$ при $j=1,2, \ldots, 2 s$ и

$$
S_{m}^{*}(f)(x)=\sum_{j=1}^{2 s}(-1)^{j} 2^{m_{j}} \int_{\Delta_{0}^{\left(m_{j}\right)}} f(t) d \mu(t)=\sum_{j=1}^{2 s}(-1)^{j} 2^{m_{j}} A_{j},
$$

где

$$
A_{j}=\int_{\Delta_{0}^{\left(m_{j}\right)}} f(t) d \mu(t)=\sum_{i=j}^{\infty} \lambda_{i+1}\left(\frac{1}{2^{m_{i}}}-\frac{1}{2^{m_{i+1}}}\right) .
$$

Если $x \in \Delta_{0}^{\left(m_{\nu-1}\right)} \backslash \Delta_{0}^{\left(m_{\nu}\right)}$ при $\nu=2 s, 2 s-1, \ldots, 1$, то $\Delta_{x}^{\left(m_{j}\right)}=\Delta_{0}^{\left(m_{j}\right)}$ при $j=1,2, \ldots, \nu-1$ и

$$
\begin{aligned}
S_{m}^{*}(f)(x) & =\sum_{j=1}^{2 s}(-1)^{j} 2^{m_{j}} \int_{\Delta_{x}^{\left(m_{j}\right)}} f(t) d \mu(t)=\sum_{j=1}^{\nu-1}+\sum_{j=\nu}^{2 s} \\
& =\sum_{j=1}^{\nu-1}(-1)^{j} 2^{m_{j}} A_{j}+\sum_{j=\nu}^{2 s}(-1)^{j} 2^{m_{j}} \lambda_{\nu} \frac{1}{2^{m_{j}}}=\sum_{j=1}^{\nu-1}(-1)^{j} 2^{m_{j}} A_{j}+\lambda_{\nu} \sum_{j=\nu}^{2 s}(-1)^{j} .
\end{aligned}
$$

Положим теперь $m_{j}=2 j$ и пусть $\lambda_{j}$ удовлетворяют условиям

1) $\left|\lambda_{j}\right| \uparrow+\infty$

2) $\lambda_{j}=\left|\lambda_{j}\right|(-1)^{j+1}$

3) $\frac{\left|\lambda_{j+1}\right|}{4^{j}} \downarrow 0$;

4) $\left|\lambda_{j+1}\right| \leqslant 2\left|\lambda_{j}\right|$

5) существуют константы $C_{1}, C_{2}>0$ такие, что $C_{1}\left|\lambda_{n}\right| n \leqslant \sum_{j=1}^{n}\left|\lambda_{j}\right| \leqslant C_{2}\left|\lambda_{n}\right| n$.

Тогда

$$
A_{j}=\frac{3}{4} \sum_{i=j}^{\infty} \frac{\lambda_{i+1}}{4^{i}}, \quad \operatorname{sgn} A_{j}=\operatorname{sgn} \lambda_{j+1}=(-1)^{j}
$$

и

$$
\frac{1}{2} \frac{\left|\lambda_{j+1}\right|}{4^{j}}<\left|A_{j}\right|<\frac{\left|\lambda_{j+1}\right|}{4^{j}}
$$

Отсюда находим, что

$$
C_{3}\left|\lambda_{2 s}\right| 2 s \leqslant\left|\sum_{j=1}^{2 s}(-1)^{j} 2^{m_{j}} A_{j}\right| \leqslant \sum_{j=1}^{2 s}\left|\lambda_{j+1}\right| \leqslant C_{4}\left|\lambda_{2 s}\right| 2 s
$$

и

$$
C_{5}\left|\lambda_{\nu}\right| \nu \leqslant\left|\sum_{j=1}^{\nu-1}(-1)^{j} 2^{m_{j}} A_{j}+\lambda_{\nu} \sum_{j=\nu}^{2 s}(-1)^{j}\right| \leqslant C_{6}\left|\lambda_{\nu}\right| \nu
$$


Положим, наконец,

$$
\left|\lambda_{k}\right|=\frac{k^{1 / q}}{\log ^{2}(k+1)} \quad\left(\frac{1}{p}+\frac{1}{q}=\alpha\right) .
$$

Тогда

$$
C_{7} \frac{n^{1 / q}}{\log ^{2} n} \leqslant\|f\|_{n} \leqslant C_{8} \frac{n^{1 / q}}{\log ^{2} n}
$$

и, значит,

$$
\sum_{n}\left(\frac{\|f\|_{n}}{n^{\alpha}}\right)^{p} \leqslant C_{9} \sum \frac{1}{\log ^{2 p} n} \frac{1}{n^{p(\alpha-1 / q)}}<\infty .
$$

Однако

$$
\begin{aligned}
\left\|S_{m}^{*}(f)\right\|_{n}^{n} & \geqslant\left(C_{3}\left|\lambda_{2 s}\right| 2 s\right)^{n}\left|\Delta_{0}^{\left(m_{2 s}\right)}\right|+\sum_{\nu=1}^{2 s}\left|\Delta_{0}^{\left(m_{\nu-1}\right)} \backslash \Delta_{0}^{\left(m_{\nu}\right)}\right|\left(C_{5}\left|\lambda_{\nu}\right| \nu\right)^{n} \\
& \geqslant 3 C_{5}^{n} \sum_{\nu=1}^{2 s} \frac{\nu^{n(1+1 / q)}}{4^{\nu} \log ^{2 n}(\nu+1)}
\end{aligned}
$$

Taк как

$$
\left(\sum_{\nu=1}^{\infty} \frac{\nu^{n(1+1 / q)}}{4^{\nu} \log ^{2 n}(\nu+1)}\right)^{1 / n} \geqslant C_{10} \frac{n^{1+1 / q}}{\log ^{2} n}
$$

TO

$$
\sup _{m} \sum\left(\frac{\left\|S_{m}^{*}(f)\right\|_{n}}{n^{\alpha+1-\varepsilon}}\right)^{p}=+\infty
$$

и теорема доказана.

\section{СПИСОК ЦИТИРОВАННОЙ ЛИТЕРАТУРЫ}

[1] Голубов Б. И., Ефимов А. В., Скворцов В. А. Ряды Уолша и преобразования Уолша. Теория и приложения. М.: Наука, 1987.

[2] Finet C., Tkebuchava G. E. Walsh-Fourier series and their generalizations in Orlicz spaces // J. Math. Anal. Appl. 1998. V. 221. P. 405-418.

[3] Канторович Л.В., Акилов Г. П. Функциональньй анализ. М.: Наука, 1977.

Саратовский государственный университет им. Н. Г. Черньшевского

Поступило

E-mail: lukomskyds@info.sgu.ru

30.01 .2001 